\title{
Review
}

\section{Subthalamic Nucleus Deep Brain Stimulation in Parkinson's Disease: The Effect of Varying Stimulation Parameters}

\author{
Viswas Dayal*, Patricia Limousin and Thomas Foltynie \\ Sobell Department of Motor Neuroscience, UCL Institute of Neurology and The National Hospital for Neurology \\ and Neurosurgery, Queen Square, London, UK
}

Accepted 19 April 2017

\begin{abstract}
Subthalamic Nucleus Deep Brain Stimulation (STN DBS) is a well-established and effective treatment modality for selected patients with Parkinson's disease (PD). Since its advent, systematic exploration of the effect of stimulation parameters including the stimulation intensity, frequency, and pulse width have been carried out to establish optimal therapeutic ranges. This review examines published data on these stimulation parameters in terms of efficacy of treatment and adverse effects. Altering stimulation intensity is the mainstay of titration in DBS programming via alterations in voltage or current settings, and is characterised by a lower efficacy threshold and a higher side effect threshold which define the therapeutic window. In addition, much work has been done in exploring the effects of frequency modulation, which may help patients with gait freezing and other axial symptoms. However, there is a paucity of data on the use of ultra-short pulse width settings which are now possible with technological advances. We also discuss current evidence for the use of novel programming techniques including directional and adaptive stimulation, and highlight areas for future research.
\end{abstract}

Keywords: Deep brain stimulation (DBS), Parkinson's Disease (PD), Subthalamic Nucleus (STN), electrical parameters

\section{INTRODUCTION}

Deep brain stimulation (DBS) of the subthalamic nucleus (STN) has been well established as an effective treatment option for selected patients with Parkinson's disease (PD) since its use was first demonstrated in humans over two decades ago [1-3]. Systematic exploration of electrical parameters and their various effects was carried out in the early era of DBS being made widely available, and included examining the effects of altering stimulation intensity, frequency, and pulse-width (PW) $[4,5]$. There

\footnotetext{
${ }^{*}$ Correspondence to: Dr. Viswas Dayal, Sobell Department of Motor Neuroscience, UCL Institute of Neurology and The National Hospital for Neurology and Neurosurgery, Box 146, Queen Square, London, WC1N3BG, UK. Tel.: +440203 4488736; Fax: +44 0203 4488642; E-mail: v.dayal@ucl.ac.uk.
}

has since been further work on alternative parameter settings, largely focusing on the effects of modulating frequency values [6-14].

In recent years, in addition to these stimulation parameters, novel programming techniques along with advances in technology have enabled more selective spatial control of stimulation current with directional steering, and the use of feedback signals to continuously and automatically adjust delivery of current as required with closed-loop adaptive stimulation methods [15-22].

Here, we review the published data to date on the effects of variation of electrical stimulation parameters including amplitude, frequency, and pulse width as it applies to subthalamic DBS in Parkinson's disease, as well as newer programming techniques of directional and adaptive stimulation. 


\section{METHODS}

A structured Pubmed search was performed through August 2016 with search terms "Subthalamic Deep Brain Stimulation in Parkinson's Disease", "voltage", "current", "frequency", "pulse width" and "side effects". We reviewed case series, observational studies, and controlled trials that examined the clinical effect of quantitatively varying one or more stimulation parameters on symptoms of Parkinson's disease, and on the occurrence of adverse effects. For novel programming techniques, we combined the first search term with "adaptive" and "directional" respectively, and included interventional or descriptive case reports, case series and controlled trials that compared these techniques to conventional DBS. Only articles focusing on subthalamic stimulation in Parkinson's disease and in English were included.

\section{THE EFFECT OF VARYING PARAMETERS}

Any positive or negative effect emerging as a result in a change in the stimulation parameters delivered through a DBS contact will depend entirely on the precise location of that contact in the brain. Given the natural anatomical variation between patients, as well as variations in surgical technique, targeting and precision, there is inevitably a confounding factor in the interpretation of stimulation adjustments between patients. Nevertheless, important lessons can be learned by systematic study of large numbers of patients, and the data emerging from such studies is presented below.

\section{Stimulation intensity}

The stimulation intensity has the greatest effect on ameliorating Parkinsonian motor signs relative to energy-equivalent changes in other parameters, and is the most commonly titrated parameter in the initial stages of subthalamic DBS programming. This can be done by controlling the voltage or the current, which are interrelated by the impedance factor. Implantable pulse generators (IPGs) programmed to use constant voltage (CV) titration control the maximum voltage associated with each pulse, while the current will vary depending on the impedance. Conversely, programming using constant current (CC) provides a specified electrical current while adjusting the voltage to compensate for the impedance [23].
In-vitro studies of constant current stimulation have demonstrated the current waveform to be more uniform in terms of intensity, exhibiting less decay than in constant-voltage stimulation. However, comparisons of $\mathrm{CC}$ and $\mathrm{CV}$ stimulation to achieve equivalent motor efficacy have not shown any significant differences in non-motor outcomes, including cognition, mood, and quality of life in a double-blind crossover trial [23]. A retrospective analysis of 19 patients with PD and dystonic syndromes switched from $\mathrm{CV}$ to $\mathrm{CC}$ stimulation reported no change in measured clinical outcomes and therapy satisfaction at 6 months [24].

Rizzone et al. carried out early systematic exploration of the impact of various parameter settings in STN DBS in a double-blinded evaluation of 10 patients. They examined the relationship of stimulus intensity in terms of current and the occurrence of therapeutic as well as adverse effects at various frequency and pulse-width settings. The intensity required to produce the clinical effect of loss of wrist rigidity ranged from 0.7 to $1.7 \mathrm{~mA}$, and the intensity required to produce adverse effects ranged from 1.3 to $3.4 \mathrm{~mA}$ [4]. The relationships between the stimulus intensity required to produce these effects and frequency as well as pulse-width are discussed in the corresponding sections below.

Subsequently, in a double blinded assessment of 12 patients, Moro and colleagues found that the mean highest tolerated voltage was $3.5 \mathrm{~V}$, while keeping frequency constant between $130-185 \mathrm{~Hz}$ and PW constant between 60-90 $\mu$ s [5]. Bradykinesia and rigidity significantly improved at $2 \mathrm{~V}$ and $3 \mathrm{~V}$ with testing carried out at $1 \mathrm{~V}$ intervals, while response to tremor was seen starting at $1 \mathrm{~V}$, with $2 \mathrm{~V}$ and $3 \mathrm{~V}$ being progressively more effective. The greatest beneficial effect on these segmental motor signs was noted to be at $3 \mathrm{~V}$, with no significant additive benefit at voltage settings above this.

Voltage-dependent side effects commonly consist of motor or sensory symptoms, occurring in the majority with progressive increase in voltage. Anatomically, these effects are thought to represent current spread to the pyramidal tract, causing muscle contractions, and the medial lemniscus, resulting in paraesthesias. Autonomic effects may include excessive sweating, flushing, mydriasis, tachycardia, and a sensation of heat or cold. In an intraoperative investigation of clinical effects produced by STN stimulation in 17 PD patients, Sauleau et al. found that using $130 \mathrm{~Hz}$ and $100 \mu$ s settings, the threshold for complete disappearance of wrist rigidity was 
$0.94 \mathrm{~V}$. The mean adverse effect thresholds were: $2 \mathrm{~V}$ for paraesthesias, $3 \mathrm{~V}$ for oculomotor effects, $3.1 \mathrm{~V}$ for autonomic effects, and $3.4 \mathrm{~V}$ for dystonic effects [25].

Tommasi et al. investigated pyramidal side effects of subthalamic stimulation by progressively increasing voltages through chronically used contacts while keeping PW and frequency constant at $60 \mu \mathrm{s}$ and $130 \mathrm{~Hz}$ respectively. Motor side effects were the most frequent, seen in 27 out of 28 electrodes, followed by sensory symptoms in 23 . The most frequent adverse effects included contractions involving the facial muscles, often affecting bilateral upper facial and contralateral lower facial muscles. Dysarthria was observed in about $25 \%$ of subjects. Oculomotor side effects were seen in 6 out of 28 electrodes tested, and most commonly consisted of reduced gaze ipsilateral to stimulation, progressing to contralateral gaze deviation with increasing voltage. The median voltage required to produce oculomotor effects was $5.5 \mathrm{~V}$, as compared to $4.5 \mathrm{~V}$ for sensory symptoms. Nausea and excessive sweating also occurred in 6 electrodes. Non-specific effects were common and nearly half of subjects experienced symptoms such as dizziness, a sense of heavy-headedness or lightheadedness, feeling of an electric current through the body, or malaise. They noted that habituation to sensory, oculomotor, and autonomic effects such as nausea and excessive sweating occurred rapidly as opposed to pyramidal tract side effects which tended to be persistent. No affective or behavioural effects related to stimulation were noted [26].

Eyelid opening apraxia has also been observed, and has been shown to have a mean threshold of occurrence of $5.2 \mathrm{v}$, although this symptom may be present as part of PD itself, and is occasionally relieved by stimulation [2, 26-28]. As well as these, stimulationinduced dyskinesias were observed in 5 of 12 patients, and foot dystonia in one of these [5].

In addition to voltage dependent adverse effects discussed, progressively increasing voltage (median $5.5 \mathrm{v}$ ) at the standard frequency of $130 \mathrm{~Hz}$ has been shown to worsen gait and increase freezing episodes, similar to the condition off-stimulation [6]. Speech intelligibility and articulation are also impaired with increasing amplitude, particularly above $3.5 \mathrm{~V}$ [29], and a stronger correlation with high voltages in the left STN and speech impairment has been observed [26, 30, 31]. Stimulation related psychiatric effects such as mania are also widely recognised, and attributed to involvement of limbic structures from stimulation of anteromedial neurons in the STN.
These often respond to either a reduction in stimulation intensity or shifting to using more dorsolateral electrode contacts [32, 33].

While quantitative data on time to habituation of adverse effects is limited, in the authors' experience, sensory and autonomic effects tend to subside within seconds if the stimulation intensity is maintained at the threshold level at which these occur. Time to habituation of capsular effects has not been explored as they are not well-tolerated.

\section{Frequency}

The use of frequencies of less than $50 \mathrm{~Hz}$ in subthalamic stimulation has been shown not to have a clinically significant effect on measurable motor signs in Parkinson's disease, even when combined with higher compensatory values of stimulus intensity and pulse-width [4]. In fact, very low frequencies of $5-10 \mathrm{~Hz}$ have been found to worsen motor symptoms, particularly bradykinesia, compared with no stimulation [5, 34, 35]. Moro et al. noted that all frequencies over $50 \mathrm{~Hz}$ significantly improved bradykinesia and tremor, while the threshold for response in terms of rigidity was $33 \mathrm{~Hz}$. The ceiling of beneficial effect was in the range of $130-185 \mathrm{~Hz}$, with progressive improvement in cardinal motor signs with increasing frequency which was significant between $50 \mathrm{~Hz}$ and $130 \mathrm{~Hz}$ but not between $130 \mathrm{~Hz}$ and $185 \mathrm{~Hz}$ or $250 \mathrm{~Hz}$ [5]. Observations have been made of requiring a lower stimulus intensity to achieve the same clinical effect with increasing frequencies between 90 and $170 \mathrm{~Hz}$, but this was not significant in a study of 10 patients [4].

Adverse effects of stimulation seem to vary depending on frequency settings: effects produced at lower frequencies $(<50 \mathrm{~Hz})$ include worsening of tremor and myoclonic jerks as opposed to paraesthesias, muscle contractions and dyskinesias at higher frequencies $(90-170 \mathrm{~Hz})$. At a given stimulus intensity, a trend to a lower side effect threshold was observed with progressively higher frequency settings, from $90 \mathrm{~Hz}$ to $170 \mathrm{~Hz}[4,5]$. Higher rates of $185 \mathrm{~Hz}$ and $250 \mathrm{~Hz}$ produced lower limb dyskinesias in one of 12 patients, and a subjective sensation of a heavy head in two of these [5].

In contrast to the good response seen in alleviation of segmental motor signs of PD with STN stimulation, gait dysfunction and other axial symptoms can evolve to become relatively refractory to conventional programming settings. High frequency 
stimulation at conventional settings of $130 \mathrm{~Hz}$ combined with high voltage has even been associated with increased incidence of gait freezing $[6,36]$. This has led to multiple studies investigating the effect of alternative frequency settings on gait. In a randomised blinded assessment, Moreau et al. examined 13 patients with severe gait disorders on frequency settings of $60 \mathrm{~Hz}$ and $130 \mathrm{~Hz}$, with voltages adjusted so that the total energy delivered was constant. While there was no significant difference in UPDRS gait and axial subscores, $60 \mathrm{~Hz}$ stimulation resulted in a significantly lower number of freezing episodes and reduction in number of steps on a Stand-Walk-Sit (SWS) test. The clinical benefit on gait persisted at 8 months follow up in $85 \%$ of patients, although they were noted to be on higher doses of levodopa at this time [6].

A randomised trial of 14 patients with doubleblind crossover design comparing $60 \mathrm{~Hz}$ and $130 \mathrm{~Hz}$ energy-equivalent stimulation found similar improvements in axial symptoms and akinesia with the lower frequency setting when assessed at one hour, with no significant differences in segmental signs between the two settings. Significant reductions in total UPDRS-III as well as axial and akinesia subscores, and a timed 10-metre walk test were noted with the lower frequency setting. Also of note in this study is that for 5 patients the optimal contact positions for low frequency stimulation were more ventrally located in the subthalamic nucleus than optimal contacts for $130 \mathrm{~Hz}$ stimulation [9].

In a case series, Xie et al. described two patients with acute worsening of freezing of gait (FOG) at standard frequency subthalamic stimulation $(130 \mathrm{~Hz})$ following new activation of their DBS systems. These demonstrated the immediate negative effect of high frequency stimulation as distinct from chronic adverse effects and those of disease progression, which complement findings in patients who have had stimulation for longer periods. They reported amelioration of FOG with $60 \mathrm{~Hz}$ stimulation and initially unchanged amplitude and pulse width settings. The effect persisted at a 10 month follow up, and was present in both ON and OFF medication states. In addition to FOG, there was noted to be improvement in bradykinesia and other axial symptoms with low frequency stimulation [36]. Subsequently, this effect on axial symptoms was reproduced with $60 \mathrm{~Hz}$ stimulation in a small randomised double-blind trial, including improvement in dysphagia and aspiration frequency specifically, quantified as a $57 \%$ reduction on a modified barium swallow assessment [10].
Another case series reported that in patients with dopa-responsive axial and gait dysfunction that initially worsened with high frequency stimulation $(130-185 \mathrm{~Hz})$, there was subsequent improvement with $60 \mathrm{~Hz}$ stimulation in four out of five patients. Immediate worsening of gait and freezing was also demonstrated on experimental switching of settings back to high frequency. Ventral contacts were used in all patients, and double monopolar configuration involving concomitant dorsal and ventral contacts was utilised in three patients due to observed beneficial effects on gait and reduction in dyskinesias [11].

However, the evidence for low frequency stimulation being beneficial for axial symptoms of PD is not unequivocal. The largest reported cohort studied involved 45 patients with refractory axial symptoms on high frequency $(130 \mathrm{~Hz})$ stimulation who were switched to $80 \mathrm{~Hz}$ settings in an open label trial, and showed no significant change in total motor UPDRS and axial and gait subscores at a median assessment period of 112 days [7]. In addition, in a randomised trial of 20 patients with gait difficulties following STN stimulation, Phibbs et al. did not find any improvement in stride length or number of freezing episodes with switching from $130 \mathrm{~Hz}$ to $60 \mathrm{~Hz}$. However, notably, other parameters in this study including the stimulation intensity were kept constant at both frequency settings, resulting in a lower total electrical energy delivered (TEED) value at low frequency [8].

Furthermore, despite the majority of studies on the utility of low frequency stimulation showing initial improvement in gait and freezing with low frequency stimulation in the range of $60-80 \mathrm{~Hz}[6$, $9-11,36]$, it is possible that these effects may not be sustained over time. An open label trial involving 11 patients found that despite initial improvements in gait as measured by performance on the SWS test after switching from $130 \mathrm{~Hz}$ to $80 \mathrm{~Hz}$ at equivalent energy delivered, the benefit was not maintained at 1, 5 and 15 months. Reduction in freezing episodes was also short-lived, being sustained at one month but not subsequently [12].

In an open label prospective analysis, Brozova et al. found mixed results with low frequency stimulation among 12 patients with gait dysfunction and postural instability; three could not tolerate low frequency due to worsening of segmental symptoms, two had worsening of postural stability and gait, while seven benefited [13].

In addition, although most studies have reported no significant change in control of segmental symptoms 
as measured by UPDRS with low frequency stimulation, it has been observed that tremor control is often worse than at standard high frequencies [10, 12, 36]. Low frequency stimulation also may not produce beneficial effects over conventional higher frequency settings in patients who do not suffer from problems with gait and postural control [14].

\section{Pulse width}

Pulse width values available for use in subthalamic stimulation in PD have conventionally ranged from $60 \mu$ s to $450 \mu \mathrm{s}$. Increasing pulse width values up to a certain level has been shown to require correspondingly lower stimulus intensities to achieve a required clinical effect. This relationship was observed by Rizzone et al. between a low pulse width of $60 \mu \mathrm{s}$ and higher values of $210 \mu \mathrm{s}$ or $450 \mu \mathrm{s}$, but not between these higher pulse width parameters, where the effect seems to plateau. A parallel relationship exists with the occurrence of adverse effects at increasing pulse widths: i.e. a lower stimulus intensity is required to produce adverse effects at higher pulse width values. This results in a narrower therapeutic window (magnitude of difference in the intensity value required to produce a clinical effect and that required to produce adverse effects) with increasing pulse widths [4].

Similarly, Moro et al. found that of the 12 patients studied, 5 were unable to tolerate PWs of greater than $210 \mu \mathrm{s}$ when combined with a voltage of $75 \%$ of the usual chronic stimulation setting. The mean highest tolerated level was $190 \mu \mathrm{s}$ in this group. Adverse effects that limited use of higher PW values were similar to those produced by increasing stimulation intensity; muscle contractions, paraesthesias, dysarthria and postural tremor were commonly observed. Improvement in tremor control and rigidity was observed at all PWs studied between $60 \mu \mathrm{s}$ and $210 \mu \mathrm{s}$, while reduction in bradykinesia was only significant at $60 \mu$ s relative to baseline [5].

There has been limited further exploration of the effects of pulse width in STN DBS beyond the pioneering data available from the work of Rizzone and Moro discussed. However, more recently, interest in the effects of PWs shorter than the conventional lower limit of $60 \mu \mathrm{s}$ has emerged, as this has now become possible with advances in DBS equipment.

Reich et al. recently reported their findings on the use of PWs of less than $60 \mu$ s at a fixed frequency of $130 \mathrm{~Hz}$ in a monopolar review session of four patients. An inverse relationship between PW and therapeutic window was noted in all subjects, with therapeutic window (TW) being defined as the difference in the minimum stimulation current required to produce adverse effects and that required to produce the clinical effect of loss of rigidity (efficacy threshold). Compared to standard $60 \mu$ s stimulation, the TW increased by a mean of $182 \%$ with a PW of $30 \mu \mathrm{s}$, and decreased by $46 \%$ with a PW of $120 \mu \mathrm{s}$. TWs could not be obtained at lower PWs of $20 \mu \mathrm{s}$ due to a lack of capsular response using a predefined maximum of $10 \mathrm{~mA}$ stimulation intensity. The corresponding stimulation current required for rigidity control increased with reducing PWs, from a mean of $1.6 \mathrm{~mA}$ at $60 \mu \mathrm{s}$ to $2.9 \mathrm{~mA}$ at $30 \mu \mathrm{s}$. Furthermore, the authors noted that while this efficacy threshold in $\mathrm{mA}$ increased at lower PWs, the total charge per pulse required for the clinical effect of rigidity control decreased. They postulated short PW may therefore potentially prolong IPG battery life [37].

Table 1 summarises the findings of studies discussed above examining the effects of varying different stimulation parameters.

\section{Directional steering}

Directional stimulation involves using segmented or multi-contact electrodes which replace conventional cylindrical electrodes, so that selective activation of one or more contacts can be used to produce a field of stimulation in the desired orientation to more selectively target adjacent neural tissue.

Intra-operative proof of concept studies to explore hypotheses that directional steering of current would result in more efficient stimulation and a reduction in adverse effects have been carried out in the last 2-3 years [15-17]. The first of these showed that using a specially designed lead with six directional contacts (3 each on 2 horizontal levels), the widest therapeutic window obtained with directional stimulation was on average $41 \%$ larger than with omnidirectional stimulation which simulated a conventional ring-shaped electrode. The therapeutic window could be increased in 10 of 11 patients compared to using omnidirectional stimulation. In addition to this, the average current threshold for therapeutic effect was noted to be $43 \%$ lower with best directional stimulation [15].

A similar intraoperative study used 32-contact electrodes to compare directional stimulation in four steering modes to conventional spherical stimulation in a double-blinded assessment of 8 patients with Parkinson's disease. For 13 of the 15 side effects 


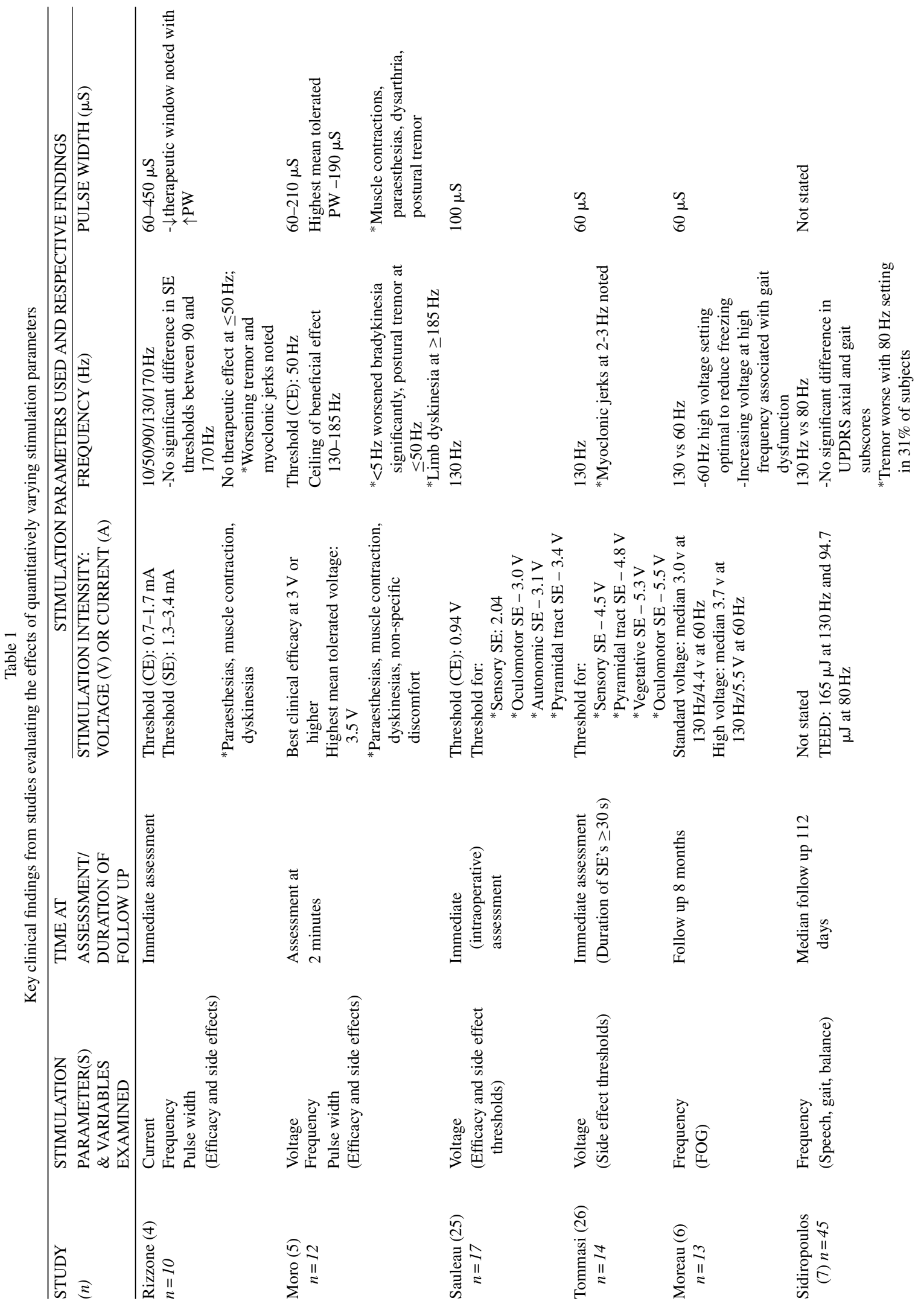




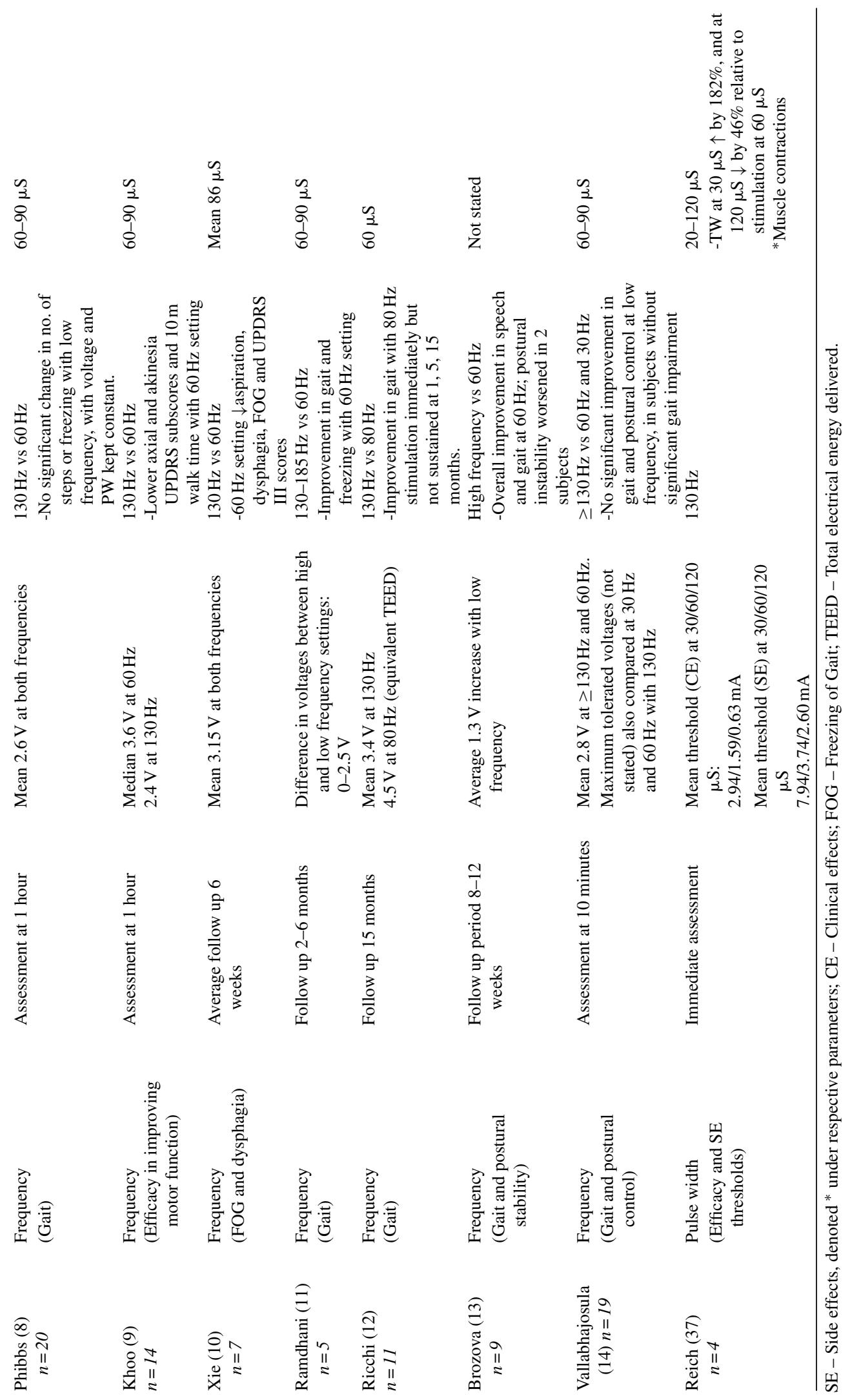


induced by conventional stimulation, steering in at least one direction led to an increase in the threshold for producing these by at least $1 \mathrm{~mA}$. The TW was evaluated with respect to improvement in rigidity as the therapeutic effect in 3 patients; all of whom demonstrated an increase of between 0.5 and $2.5 \mathrm{~mA}$ with directional stimulation [16].

Following the results of intraoperative studies, Steigerwald et al. reported their clinical experience with implantation of the $\mathrm{CE}$ marked Vercise $P C$ (Boston scientific $^{\mathrm{TM}}$ ) directional system with multiple independent current source control (MICC) in 7 patients with PD. A post-operative monopolar review session was used to determine thresholds for therapeutic and adverse effects, and expansion in the TW with directional modulation seen in previous experimental studies was confirmed at 17 of 22 stimulation sites. This study was designed to demonstrate feasibility rather than efficacy, and at a median of 4 months follow up, all patients remained on directional stimulation without needing to switch to conventional ring mode stimulation [18].

As expected, the direction of stimulation generally correlates with adverse effects produced by activation of known fibre tracts at those locations, with lateral stimulation producing the most common adverse effects of focal muscle contraction and dysarthria from corticospinal tract activation $[15,16,18]$.

\section{Adaptive stimulation}

The concept of adaptive stimulation broadly involves a means of obtaining feedback on pathological brain activity and responding with varying stimulation accordingly. This has been studied with closed-loop systems which record local field potentials (LFPs) via stimulation electrodes, and in particular using beta frequency $(13-35 \mathrm{~Hz})$ activity in the STN which has been shown to correlate with the degree of motor impairment in PD [21, 38-42]. Little et al. first demonstrated the utility of this approach systematically in patients with PD in 2013. Data was collected on eight patients with unilateral STN stimulation, and they reported that compared to the unstimulated state, there was a mean reduction of $50 \%$ in UPDRS motor scores on blinded assessments with the adaptive approach compared to $31 \%$ with continuous stimulation. Not only was there more effective overall control of motor symptoms, but adaptive DBS seemed to have less than half the energy requirements of continuous stimulation by virtue of its intermittent nature [19].
This was studied again by these authors with bilateral stimulation, with independent sensing and activation on each side according to the amplitude of beta activity at the electrode. Although there was no direct comparison with continuous DBS, UPDRS motor scores were $43 \%$ better relative to no stimulation, despite stimulation being on for only $45 \%$ of the time, and resulting in an energy saving similar to that found in the first study. In addition to this, adaptive DBS seemed to have a synergistic effect with Levodopa on Parkinsonian motor signs, with an appropriate reduction in stimulation in response to reduced beta activity and clinical improvement which temporally corresponded with the action of Levodopa after its administration [20].

However, despite the encouraging findings of adaptive DBS use in the early post-operative period, as yet data is restricted to limited testing over short time periods only. There has been one case report of the beneficial effects of adaptive DBS in a freely mobile patient being maintained at day 6 post-operatively with a testing period of 2 hours. The patient was found to have stable control of segmental symptoms without dyskinesias, and also demonstrated similar improvement in axial symptoms compared to conventional continuous DBS [22].

Bour et al. combined elements of adaptive and directional stimulation by using feedback obtained with measurement of $\beta$-band power from various channels at different depths and directions using a 32 contact DBS lead. Despite a small sample, they demonstrated that stimulation in the direction of highest $\beta$-band activity in the range of $18.5-30 \mathrm{~Hz}$ was best associated with achieving the clinical outcome of reducing rigidity [17].

\section{DISCUSSION}

Control of stimulation intensity through titration of current or voltage has been the mainstay of DBS programming since its inception. However, this has limitations due to adverse effects with increasing stimulation, and the roles of altering other parameters for specific indications or to reduce adverse effects are becoming clearer as the effects of alternative settings are explored. This has been the case particularly for frequency modulation, with substantial evidence for improvement in FOG and other axial symptoms with the use of lower settings in the range of $60-80 \mathrm{~Hz}[6,9-11,36]$. The persistence of this effect however, is not certain based on current data, 
with some suggestion that it may not be maintained in the long term [12]. It is not clear whether this is due to wearing off and loss of efficacy of stimulation or natural disease progression counteracting the benefit over time. Moreover, amongst the studies that demonstrate an improvement in axial symptoms with lower frequency settings, this seems to be the case for a subset of patients; it has been observed that those who do not have tremor-dominant PD and have symptoms of gait dysfunction with high frequency stimulation are more likely to benefit $[12,13]$. It is also interesting that Xie et al. demonstrated in a small randomised trial that the effect extends specifically to swallowing function, with a substantial reduction in aspiration [10]. This important finding may broaden the applicability of lower frequency stimulation to PD patients treated with DBS who suffer from the wider range of axial symptoms including dysphagia rather than just those with gait dysfunction.

It is also worth noting that the use of UPDRS axial and gait subscores in many studies to measure outcomes may have resulted in diminished detection of effect than if more sensitive tools such as rapid $360^{\circ}$ turns and the addition of dual-tasking were used to assess freezing of gait [43, 44]. The mechanism of low frequency stimulation and its beneficial effect on axial symptoms in selected patients is not clear. One of the proposed explanations is spread of current to neurons projecting to the pedunculopontine nucleus (PPN) which is in $5 \mathrm{~mm}$ proximity to the STN and has reciprocal connections with it, as low frequency stimulation of this structure directly and in combination with STN stimulation has been shown to improve FOG [45-48]. Relatively greater reduction in akinesia noted with lower frequency stimulation has also been suggested as a possible mechanism for improvement in FOG [6, 9, 12]. It has been postulated that the negative impact on gait with high frequency settings may be due to a change in STN function in advanced PD caused by direct high frequency stimulation, particularly when combined with high voltages, as this is likely to result in current diffusion into surrounding structures [6]. The location of contacts in the STN may also influence the effectiveness of low frequency stimulation, with ventrally located contacts being favourable as demonstrated by Khoo and colleagues [9]. This may also be partly responsible for the heterogeneity of data on the effectiveness of low frequency stimulation, as many of the studies that demonstrated a significant improvement tended to have largely used ventrally located contacts $[6,9]$. It should also be noted that most studies that failed to show at least an initial improvement with low frequency stimulation in patients who had troublesome axial symptoms used lower TEED values at low frequency stimulation $[7,8]$.

While a reasonable amount of work has been done on the use of alternative frequency settings, larger prospective blinded trials with detailed gait assessments and longer follow up periods are required to confirm the benefit of low frequency settings for axial symptoms in the long term, and further define phenotypic subsets of patients who may benefit most.

In contrast, there is a paucity of data on the use of alternative pulse width settings, in part due to the lack of availability of PWs shorter than $60 \mu$ s until relatively recently in DBS systems. Only selected manufacturers are now producing devices with this capability for use with new implants. The effect of ultra-short PWs utilised by Reich and colleagues using the Boston Scientific Vercise ${ }^{\mathrm{TM}}$ system is very encouraging in demonstrating the potential for significant increases in the therapeutic window with this approach. This is thought to be mediated by more selective action of stimulation on neural fibre tracts responsible for relief of symptoms while avoiding those such as corticospinal and corticobulbar fibres that result in adverse effects. This has been demonstrated previously by Groppa et al. in patients with essential tremor, where using strength-duration curves plotted using various $\mathrm{PW}$ values, the chronaxie (measure of excitability of neural elements) calculated for suppression of tremor was shown to be significantly different to that for induction of ataxia, with values of $27 \mu \mathrm{s}$ and $52 \mu \mathrm{s}$ respectively. They concluded that a stimulation PW closer to the chronaxie of tremor suppression would provide a wider therapeutic window between tremor relief and induction of side effects [49]. The same concept was used by Reich et al. to derive a strength-duration plot model for axons of different diameters, showing a divergence of action potential thresholds at lower PWs [37]. While the exact nature of the fibres responsible for rigidity control is not known, the magnitude of effect on the TW with short PW stimulation found in these patients certainly merits further investigation. Larger randomised blinded trials to confirm the effects on pyramidal as well as other common adverse effects of stimulation such as dysarthria and axial symptoms need to be carried out, as this has the potential to have a significant clinical impact on existing and new DBS patients.

As has been stated earlier, in interpreting the clinical effects of varying stimulation parameters based 
on the available data, one should bear in mind that most of the reviewed studies do not systematically include the effect of the exact location of electrodes or contacts used in their analyses. Further randomised blinded trials controlling for this variable will improve comparability between subjects and studies.

Newer techniques have added a further dimension to programming using basic parameters by modulation of stimulation in space and time using directional and adaptive stimulation respectively. While still in early stages, available data suggests not only a reduction in side effects, but significant improvements in energy requirements in terms of therapeutic current required. Short pulse width, adaptive stimulation and directional steering all share the same goal of improving specificity of stimulation to appropriate neural elements to achieve the above clinical effects. While systems capable of directional stimulation and short pulse width have been made commercially available recently, technology is lagging behind in providing devices capable of adaptive programming. Indeed, it would be of interest to examine whether a synergistic or cumulative effect resulting from combining all of these programming techniques exists. However, two decades on, the need for more robust data on the use of simple parameters such as shorter pulse widths is long overdue.

\section{CONFLICT OF INTEREST}

The authors have no conflict of interest to report.

\section{REFERENCES}

[1] Limousin P, Pollak P, Benazzouz A, Hoffmann D, Le Bas J, Perret J, Benabid A, \& Broussolle E (1995) Effect on parkinsonian signs and symptoms of bilateral subthalamic nucleus stimulation. Lancet, 345, 91-95.

[2] Limousin P, Krack P, Pollak P, Benazzouz A, Ardouin C, Hoffmann D, \& Benabid A (1998) Electrical stimulation of the subthalamic nucleus in advanced Parkinson's disease. N Engl J Med, 339, 1105-1111.

[3] Weaver FM (2009) Bilateral deep brain stimulation vs best medical therapy for patients with advanced Parkinson disease: A randomized controlled trial. JAMA, 301, 63.

[4] Rizzone M (2001) Deep brain stimulation of the subthalamic nucleus in Parkinson's disease: Effects of variation in stimulation parameters. J Neurol Neurosurg Psychiatry, 71, 215-219.

[5] Moro E, Esselink R, Xie J, Hommel M, Benabid A, \& Pollak $P$ (2002) The impact on Parkinson's disease of electrical parameter settings in STN stimulation. Neurology, 59, 706713.

[6] Moreau C, Defebvre L, Destee A, Bleuse S, Clement F, Blatt J, Krystkowiak P, \& Devos D (2008) STN-DBS frequency effects on freezing of gait in advanced Parkinson disease. Neurology, 71, 80-84.

[7] Sidiropoulos C, Walsh R, Meaney C, Poon Y, Fallis M, \& Moro E (2013) Low-frequency subthalamic nucleus deep brain stimulation for axial symptoms in advanced Parkinson's disease. J Neurology, 260, 2306-2311.

[8] Phibbs F, Arbogast P, \& Davis T (2013) 60-Hz frequency effect on gait in Parkinson's disease with subthalamic nucleus deep brain stimulation. Neuromodulation Technol Neural Interface, 17, 717-720.

[9] Khoo H, Kishima H, Hosomi K, Maruo T, Tani N, Oshino S, Shimokawa T, Yokoe M, Mochizuki H, Saitoh Y, \& Yoshimine T (2014) Low-frequency subthalamic nucleus stimulation in Parkinson's disease: A randomized clinical trial. Mov Disord, 29, 270-274.

[10] Xie T, Vigil J, MacCracken E, Warnke P, \& Kang U (2015) Low-frequency stimulation of STN-DBS reduces aspiration and freezing of gait in patients with PD. Neurology, 85, 557-557.

[11] Ramdhani R, Patel A, Swope D, \& Kopell B (2015) Early use of $60 \mathrm{~Hz}$ frequency subthalamic stimulation in Parkinson's disease: A case series and review. Neuromodulation Technol Neural Interface, 18, 664-669.

[12] Ricchi V, Zibetti M, Angrisano S, Merola A, Arduino N, Artusi C, Rizzone M, Lopiano L, \& Lanotte M (2012) Transient effects of $80 \mathrm{~Hz}$ stimulation on gait in STN DBS treated PD patients: A 15 month follow-up study. Brain Stimul, 5, 388-392.

[13] Brozova H, Barnaure I, Alterman RL, \& Tagliati M (2009) STN-DBS frequency effects on freezing of gait in advanced Parkinson disease. Neurology, 72, 770; author reply 770771.

[14] Vallabhajosula S, Haq I, Hwynn N, Oyama G, Okun M, Tillman M, \& Hass C (2015) Low-frequency versus highfrequency subthalamic nucleus deep brain stimulation on postural control and gait in Parkinson's disease: A quantitative study. Brain Stimul, 8, 64-75.

[15] Pollo C, Kaelin-Lang A, Oertel M, Stieglitz L, Taub E, Fuhr P, Lozano A, Raabe A, \& Schupbach M (2014) Directional deep brain stimulation: An intraoperative double-blind pilot study. Brain, 137, 2015-2026.

[16] Contarino M, Bour L, Verhagen R, Lourens M, de Bie R, van den Munckhof P, \& Schuurman P (2014) Directional steering: A novel approach to deep brain stimulation. Neurology, 83, 1163-1169.

[17] Bour L, Lourens M, Verhagen R, de Bie R, van den Munckhof P, Schuurman P, \& Contarino M (2015) Directional recording of subthalamic spectral power densities in Parkinson's disease and the effect of steering deep brain stimulation. Brain Stimul, 8, 730-741.

[18] Steigerwald F, Müller L, Johannes S, Matthies C, \& Volkmann J (2016) Directional deep brain stimulation of the subthalamic nucleus: A pilot study using a novel neurostimulation device. Mov Disord, 31, 1240-1243.

[19] Little S, Pogosyan A, Neal S, Zavala B, Zrinzo L, Hariz M, Foltynie T, Limousin P, Ashkan K, FitzGerald J, Green A, Aziz T, \& Brown P (2013) Adaptive deep brain stimulation in advanced Parkinson disease. Ann Neurol, 74, 449-457.

[20] Little S, Beudel M, Zrinzo L, Foltynie T, Limousin P, Hariz M, Neal S, Cheeran B, Cagnan H, Gratwicke J, Aziz TZ, Pogosyan A, \& Brown P (2016) Bilateral adaptive deep brain stimulation is effective in Parkinson's disease. $\mathrm{J} \mathrm{Neu}$ rol Neurosurg Psychiatry, 87, 717-721.

[21] Beudel M, \& Brown P (2016) Adaptive deep brain stimulation in Parkinson's disease. Parkinsonism Relat Disord, 22, S123-S126. 
[22] Rosa M, Arlotti M, Ardolino G, Cogiamanian F, Marceglia S, Di Fonzo A, Cortese F, Rampini P, \& Priori A (2015) Adaptive deep brain stimulation in a freely moving parkinsonian patient. Move Disord, 30, 1003-1005.

[23] Ramirez de Noriega F, Eitan R, Marmor O, Lavi A, Linetzky E, Bergman H, \& Israel Z (2015) Constant current versus constant voltage subthalamic nucleus deep brain stimulation in Parkinson's disease. Stereotact Funct Neurosurg, 93, 114121.

[24] Preda F, Cavandoli C, Lettieri C, Pilleri M, Antonini A, Eleopra R, Mondani M, Martinuzzi A, Sarubbo S, Ghisellini G, Trezza A, Cavallo MA, Landi A, \& Sensi M (2016) Switching from constant voltage to constant current in deep brain stimulation: A multicenter experience of mixed implants for movement disorders. Eur J Neurol, 23, 190-195.

[25] Sauleau P, Raoul, S, Lallement F, Rivier I, Drapier S, Lajat Y, $\&$ Verin M (2005) Motor and non-motor effects during intraoperative subthalamic stimulation for Parkinson's disease. J Neurol, 252, 457-464.

[26] Tommasi G, Krack P, Fraix V, Le Bas J, Chabardes S, Benabid A, \& Pollak P (2008) Pyramidal tract side effects induced by deep brain stimulation of the subthalamic nucleus. J Neurol Neurosurg Psychiatry, 79, 813-819.

[27] Tommasi G, Krack P, Fraix V, \& Pollak P (2012) Effects of varying subthalamic nucleus stimulation on apraxia of lid opening in Parkinson's disease. J Neurol, 259, 1944-1950.

[28] Baizabal-Carvallo JF, \& Jankovic J (2016) Movement disorders induced by deep brain stimulation. Parkinsonism Relat Disord, 25, 1-9.

[29] Törnqvist AL, Schalén L, \& Rehncrona S (2004) Effects of different electrical parameter settings on the intelligibility of speech in patients with Parkinson's disease treated with subthalamic deep brain stimulation. Mov Disord, 20, 416423.

[30] Tripoliti E, Zrinzo L, Martinez-Torres I, Frost E, Pinto S, Foltynie T, Holl E, Petersen E, Roughton M, Hariz MI, \& Limousin P (2010) Effects Of subthalamic stimulation on speech of consecutive patients with Parkinson disease. Neurology, 76, 80-86.

[31] Tripoliti E, Limousin P, Foltynie T, Candelario J, AvilesOlmos I, Hariz MI, \& Zrinzo L (2014), Predictive factors of speech intelligibility following subthalamic nucleus stimulation in consecutive patients with Parkinson's disease. Mov Disord, 29, 532-538.

[32] Greenhouse I, Gould S, Houser M, Hicks G, Gross J, \& Aron A (2011) Stimulation at dorsal and ventral electrode contacts targeted at the subthalamic nucleus has different effects on motor and emotion functions in Parkinson's disease. Neuropsychologia, 49, 528-534.

[33] Chopra A, Tye S, Lee K, Sampson S, Matsumoto J, Adams A, Klassen B, Stead M, Fields J, \& Frye M (2012) Underlying neurobiology and clinical correlates of mania status after subthalamic nucleus deep brain stimulation in Parkinson's disease: A review of the literature. J Neuropsychiatry Clin Neurosci, 24, 102-110.

[34] Eusebio A, Chen C, Lu C, Lee S, Tsai C, Limousin P, Hariz M, \& Brown P (2008) Effects of low-frequency stimulation of the subthalamic nucleus on movement in Parkinson's disease. Exp Neurol, 209, 125-130.

[35] Timmermann L, Wojtecki L, Gross J, Lehrke R, Voges J, Maarouf M, Treuer H, Sturm V, \& Schnitzler A (2004) TenHertz stimulation of subthalamic nucleus deteriorates motor symptoms in Parkinson's disease. Mov Disord, 19, 13281333.

[36] Xie T, Kang U, Warnke P (2012) Effect of stimulation frequency on immediate freezing of gait in newly activated STN DBS in Parkinson's disease. J Neurol Neurosurg Psychiatry, 83, 1015-1017.

[37] Reich M, Steigerwald F, Sawalhe A, Reese, R, Gunalan K, Johannes S, Nickl R, Matthies C, McIntyre C, \& Volkmann J (2015) Short pulse width widens the therapeutic window of subthalamic neurostimulation. Ann Clin Transl Neurol, 2, 427-432.

[38] Little S, \& Brown P (2012) What brain signals are suitable for feedback control of deep brain stimulation in Parkinson's disease? Ann N Y Acad Sci, 1265, 9-24.

[39] Kühn A, Kupsch A, Schneider G, \& Brown P (2006) Reduction in subthalamic $8-35 \mathrm{~Hz}$ oscillatory activity correlates with clinical improvement in Parkinson's disease. Eur $J$ Neurosci, 23, 1956-1960.

[40] Ray N, Jenkinson N, Wang S, Holland P, Brittain J, Joint C, Stein J, \& Aziz T (2008) Local field potential beta activity in the subthalamic nucleus of patients with Parkinson's disease is associated with improvements in bradykinesia after dopamine and deep brain stimulation. Exp Neurol, 213, 108-113.

[41] Weinberger M, Mahant N, Hutchison W, Lozano A, Moro E, Hodaie M, Lang A, \& Dostrovsky J (2006) Beta oscillatory activity in the subthalamic nucleus and its relation to dopaminergic response in Parkinson's disease. J Neurophysiol, 96, 3248-3256.

[42] Kühn A, Tsui A, Aziz T, Ray N, Brücke C, Kupsch A, Schneider G, \& Brown P (2009) Pathological synchronisation in the subthalamic nucleus of patients with Parkinson's disease relates to both bradykinesia and rigidity. Exp Neurol, 215, 380-387.

[43] Snijders AH, Haaxma CA, Hagen YJ, Munneke M, \& Bloem BR (2012) Freezer or non-freezer: Clinical assessment of freezing of gait. Parkinsonism Relat Disord, 18, 149-154.

[44] Nonnekes J, Janssen AM, Mensink SH, Oude Nijhuis LB, Bloem BR, \& Snijders AH (2014) Short rapid steps to provoke freezing of gait in Parkinson's disease. J Neurol, 261, 1763-1767.

[45] Mazzone P, Lozano A, Stanzione P, Galati S, Scarnati E, Peppe A, \& Stefani A (2005) Implantation of human pedunculopontine nucleus: A safe and clinically relevant target In Parkinson's disease. Neuroreport, 16, 1877-1881.

[46] Golestanirad L, Elahi B, Graham SJ, Das S, \& Wald LL (2015) Efficacy And Safety Of Pedunculopontine Nuclei (PPN) Deep brain stimulation in the treatment of gait disorders: A meta-analysis of clinical studies. Can J Neurol Sci, 43, 120-126.

[47] Windels F, Thevathasan W, Silburn P, \& Sah P (2015) Where and what is the PPN and what is its role in locomotion? Brain, 138, 1133-1134.

[48] Stefani A, Lozano AM, Peppe A, Stanzione P, Galati S, Tropepi D, Pierantozzi M, Brusa L, Scarnati E, \& Mazzone P (2007) Bilateral deep brain stimulation of the pedunculopontine and subthalamic nuclei in severe Parkinson's disease. Brain, 130(Pt 6), 1596-1607.

[49] Groppa S, Herzog J, Falk D, Riedel C, Deuschl G. \& Volkmann J (2013) Physiological and anatomical decomposition of subthalamic neurostimulation effects in essential tremor. Brain, 137, 109-121. 